\title{
Potencial de Copaifera langsdorffii Desf. (Fabaceae) como indicadora de mudanças climáticas por meio da análise dos anéis de crescimento, Paraná, Brasil
}

\author{
Copaifera langsdorffii Desf. (Fabaceae) potential as an indicator of climate change by tree ring \\ analysis, Parana, Brazil
}

\section{Bruno Palka Miranda ${ }^{1 *}$, Victor Hugo Ferreira Andrade ${ }^{2}$, Paulo Cesar Botosso ${ }^{3}$, Tomaz Longhi Santos ${ }^{1}$, Jaçanan Eloisa de Freitas Milani ${ }^{4}$ e Carlos Vellozo Roderjan ${ }^{1}$}

${ }^{1}$ Universidade Federal do Paraná, Curitiba, PR, Brasil. *Autor para correspondência: brunopalka@gmail.com.

${ }^{2}$ Universidade Estadual do Centro Oeste, Irati, PR, Brasil.

${ }^{3}$ Empresa Brasileira de Pesquisa Agropecuária, Colombo, PR, Brasil.

${ }^{4}$ Universidade do Estado de Santa Catarina, Lages, SC, Brasil.

Submissão: 25/10/2017 | Aceite: 01/03/2019

\begin{abstract}
RESUMO
No âmbito do atual tema das mudanças climáticas, ter acesso a ferramentas que permitam a sua análise torna-se essencial para a correta compreensão de tais alterações. A interpretação dos sinais climáticos evidenciados nos anéis de crescimento das árvores é uma das maneiras de entender essas relações. Portanto, este trabalho teve como objetivo avaliar como as relações climáticas regionais tem efeito sobre os anéis de crescimento de Copaifera langsdorffii, espécie típica das florestas estacionais semideciduais do Paraná. Foram utilizadas quatro árvores provenientes da supressão da vegetação para formação do reservatório da Usina Hidrelétrica Jayme Canet Júnior, na porção média do rio Tibagi, entre os municípios de Telêmaco Borba e Ortigueira. A partir dos discos transversais de madeira, foram traçados 40 raios com o intuito de identificar e demarcar os limites dos anéis de crescimento. Posteriormente, a largura dos anéis de crescimento foi mensurada, realizando-se o controle de qualidade destas medições e a construção de uma cronologia regional para a espécie. Os dados climáticos utilizados para as correlações foram obtidos junto aos institutos oficiais de meteorologia do Paraná e junto à um banco de dados climatológico global. A cronologia gerada compreende um período de 240 anos, com intercorrelação significativa $(r=0,469$; $p<0,01)$. Foram identificadas as relações dos picos de desenvolvimento com os eventos de El Niño, onde apresentaram correlações positivas a partir da década de 1970, especialmente no ano de 1983 (El Niño mais forte atuante na região Sul). Dessa maneira, os resultados preliminares reforçam o potencial de utilização desta espécie para estudos dendroecológicos podendo, inclusive, auxiliar no entendimento das mudanças climáticas, e como se dão os seus efeitos a nível regional e local, quando incorporados a um universo amostral com maior representatividade.
\end{abstract}

PALAVRAS-CHAVE: dendrocronologia, dendroclimatologia, El Niño, pulsos de inundação, copaíba.

\section{ABSTRACT}

Under the current issue of climate change, having access to tools that allow its analysis has become essential for the correct understanding of such changes. The interpretation of climatic signals evidenced in tree rings is one of the ways to understand these relations. Therefore, this study aimed to evaluate how regional climatic relationships have an effect on the Copaifera langsdorffii growth rings, a typical species of the seasonal semideciduous forests of Parana. We used four trees as a result of the vegetation suppression to form the reservoir of the Jayme Canet Júnior Hydroelectric Power Plant, in the middle portion of the Tibagi River, between the municipalities of Telemaco Borba and Ortigueira. From the cross sections of the trees, 40 series were drawn in order to identify and demarcate the limits of the growth rings. Then, we measured the width of the growth rings and performed a quality control and the construction of a regional chronology for the species. The climatic data used for these correlations were obtained from the official meteorological institutes of Parana and a global climate database. The chronology has 240 years, with significant intercorrelation $(r=0,469, p<0.01)$. The relationship between development peaks and $E l$ Niño events was identified, with positive correlations from the 1970s, especially in 1983 (strongest El Niño in Southern Brazil). In this way, the preliminary results reinforce this species potential to be used for dendroecological studies, which may also help in the understanding of climatic changes, as well as their 
effects at a regional and local level, when incorporated into a sample universe of greater representativity.

KEYWORDS: dendrochronology, dendroclimatology, El Niño, flood pulses, copaíba.

\section{INTRODUÇÃO}

Dentre as diferentes formações florestais no Brasil, a Floresta Estacional Semidecidual tem destaque pela característica de queda das folhas das árvores em até $50 \%$ dos seus indivíduos (RODERJAN et al. 2002), e a presença de algumas espécies perenes de elevado porte e dominância, como a Copaifera langsdorffii Desf. (Fabaceae Caesalpinioideae), que alcança imponentes $35 \mathrm{~m}$ de altura e $1 \mathrm{~m}$ de diâmetro à altura do peito (CARVALHO 2003).

Estudos comprovam que nesta tipologia florestal a atividade cambial para a $C$. langsdorffii é mais intensa nos períodos de chuvas, em detrimento aos períodos de seca (LISI et al. 2008). A delimitação das camadas de crescimento se dá principalmente pela presença de faixas de parênquima axial com mais de três células de largura, contendo, em sua maioria, canais axiais normais (MARCATI et al. 2011). Os anéis de crescimento da espécie são classificados como distintos (INSIDEWOOD 2004), com porosidade difusa e predominância de vasos solitários, com camadas de crescimento individualizadas pelo parênquima axial marginal (MARCATI et al. 2011, MELO JR. et al. 2011). Diversos são os estudos que analisam a anatomia da madeira (TOMAZELLO FILHO et al. 2004, MARCATI et al. 2011, MELO JR. et al. 2011, CALLADO et al. 2014), e que reafirmam o caráter anual de formação dos anéis de crescimento. (LISI et al. 2008, COSTA et al. 2015).

A formação dos anéis de crescimento, em muitas espécies brasileiras, não está condicionada apenas pelas alterações nas taxas de precipitação, mas também em temperatura e até mesmo outras variáveis meteorológicas - umidade do ar, fotoperíodo, por exemplo (SCHÖNGART et al. 2017). Ainda, não são apenas os eventos meteorológicos de menor escala que podem influenciar o comportamento de crescimento de uma espécie. Fenômenos de maior escala, como os eventos globais de alteração climática, também podem ter efeito sobre o crescimento das árvores, ainda que seu efeito não seja perfeitamente compreendido (SCHÖNGART et al. 2004, COELHO et al. 2012).

O mais famoso e de grandes proporções é conhecido como ENSO (El Niño - Southern Oscillation, ou El Niño - Oscilação Sul (ENOS), em português), e representa o aquecimento anormal da temperatura das águas superficiais e subsuperficiais do Oceano Pacífico equatorial, além de alterações nas correntes de circulação dos ventos alísios. Com o aquecimento do oceano e redução dos ventos, começam a ser observadas mudanças da circulação da atmosfera nos níveis baixos e altos, determinando mudanças nos padrões de transporte de umidade e, portanto, variações na distribuição das chuvas em regiões tropicais e de latitudes médias e altas (OLIVEIRA 2001).

É conhecido que a região Sul do Brasil, assim como em outras regiões do continente, está ou é periodicamente sujeita a influência dos eventos ENSO. A oscilação anual da precipitação na região Sul do Brasil é bastante influenciada pelo evento El Niño e, em anos de forte atuação, a região sul e sudeste sofre com excesso de chuvas tendo a primavera como a estação que mais é afetada por esse fenômeno (CUNHA et al. 2011).

Neste sentido, estudos tem comprovado que anos sob forte influência de mudanças de temperatura e pressão da superfície dos Oceanos, principalmente Atlântico, resultam em diminuição da largura dos anéis de crescimento, bem como a influência no incremento da área basal de indivíduos arbóreos (WORBES et al. 2003, SCHÖNGART et al. 2015, LOCOSSELLI et al. 2016a). Ainda, os indivíduos arbóreos que estão sob influência dos pulsos de inundação dos rios também têm seu crescimento reduzido (SCHÖNGART et al. 2002, DEZZEO et al. 2003). Dessa forma os resultados obtidos pela dendroclimatologia mostram que o crescimento de alguns indivíduos arbóreos é sensível às condições climáticas que incidem sob os mesmos, podendo ser identificado em seus anéis de crescimento (GONÇALVES 2008, LORENSI 2012).

A escassez de informações referentes ao clima em tempos passados ou o registro de intervenções naturais/antrópicas em florestas, faz da análise dos anéis de crescimento uma ferramenta de grande auxílio, em tempos em que se buscam informações sobre as mudanças climáticas (LONGHI-SANTOS 2017). Assim, este trabalho teve por objetivos analisar macroscopicamente os limites dos anéis de crescimento de C. langsdorffii, determinar a idade das árvores selecionadas, correlacionar com fatores climáticos de grande escala, a nível global (ENSO e SST - Sea Surface Temperature ou Temperatura da Superfície do Mar (TSM), em português) e a nível regional (precipitação, temperatura e pulsos de inundação), visando compreender o comportamento de crescimento da espécie. 


\section{MATERIAL E MÉTODOS}

\section{Área de estudo}

A área de estudo localiza-se entre os municípios de Telêmaco Borba e Ortigueira, no estado do Paraná, aproximadamente entre as coordenadas $24^{\circ} 07^{\prime} \mathrm{S}$ e $50^{\circ} 42^{\prime} \mathrm{W}$, no segundo planalto paranaense. A região de Telêmaco Borba encontra-se na região média da Bacia do rio Tibagi, compreendendo uma zona formada pela Floresta Estacional Semidecidual e a Floresta Ombrófila Mista (IBGE 2012, MAACK 2012).

O clima da região é do tipo $\mathrm{Cfb}$ - mesotérmico subtropical úmido com verões quentes, segundo a classificação de Köppen (ALVARES et al. 2013). As médias térmicas anuais variam de 18 a $20^{\circ} \mathrm{C}$, atingindo valores médios de 23 a $25^{\circ} \mathrm{C}$ nos meses mais quentes e de 13 a $15^{\circ} \mathrm{C}$ nos mais frios (INMET 2015). A região apresenta uma média anual de precipitação de $1.690 \mathrm{~mm}$, com chuvas relativamente bem distribuídas ao longo do ano, sem uma estação seca e de déficit hídrico bem definida, ainda que as menores taxas médias de precipitação sejam observadas no outono/inverno. (Figura 1). A área apresenta solos classificados como Argissolos Vermelho-Amarelo, enquanto nas encostas, como Cambissolos Háplicos associados a Neossolos Litólicos (ADENESKY-FILHO 2014).

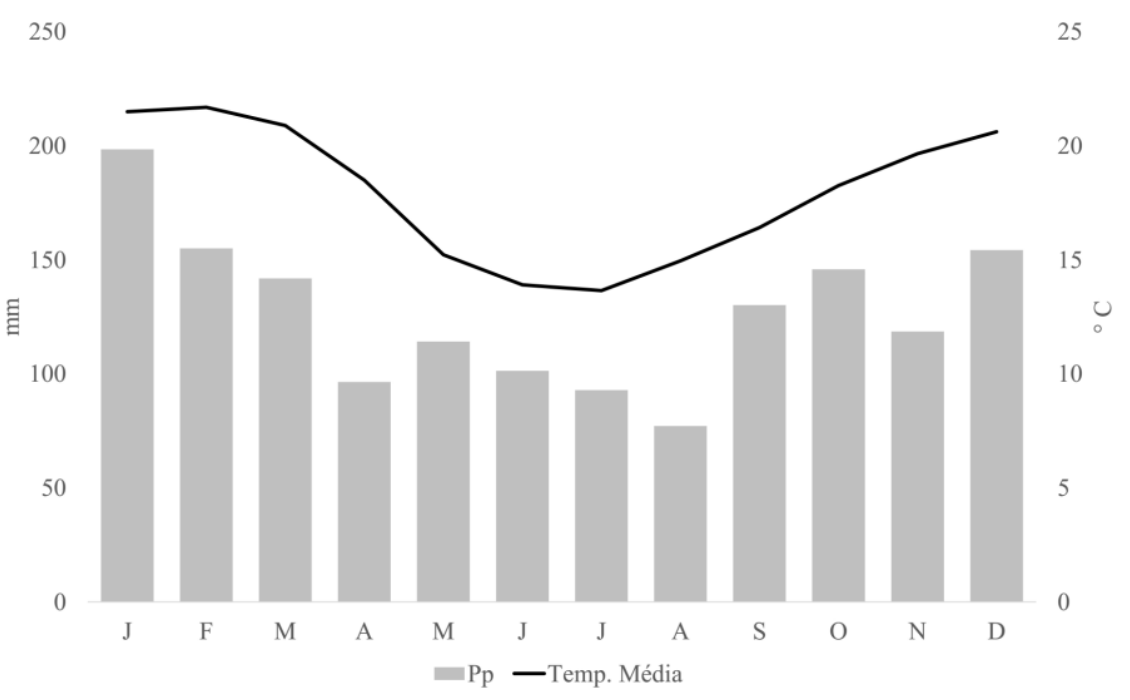

Figura 1. Série temporal de 1946-2010 de temperaturas médias (linha preta) e precipitação (barras cinza), para a região de estudo.

Figure 1. Time series from 1946-2010 average temperatures (black line) and precipitation (grey bars), for the studied area.

\section{Coleta de dados}

No ano de 2010, parte da dinâmica fluvial do trecho médio do rio Tibagi foi alterada em função da construção da Usina Hidrelétrica Jayme Canet Júnior, um consórcio entre a Companhia Paranaense de Energia Ltda. (COPEL) e a Agência Nacional de Energia Elétrica (ANEEL). Com vistas a formação do reservatório foi removida via supressão da vegetação uma área de aproximadamente 2.675 ha, por meio da Licença de Operação do Instituto Ambiental do Paraná (IAP) sob o no 27431. Foram selecionadas quatro árvores da espécie provenientes das áreas de supressão da vegetação, na planície de inundação, em área com constante interferência do rio Tibagi, sobre uma condição de hidromorfia dos solos associados.

A coleta das amostras de madeira foi realizada entre os meses de junho e setembro de 2011, através do método destrutivo, utilizando-se motosserra para obtenção dos discos de madeira (secção transversal do tronco) a aproximadamente $0,4 \mathrm{~m}$ do nível do solo, visando a obtenção de amostras com a maior representatividade possível de anéis de crescimento.

Os discos foram submetidos a secagem natural à temperatura ambiente e pelo processo de polimento superficial com lixas de diferentes granulometrias (80-600) (STOKES \& SMILEY 1968). A descrição macroscópica dos anéis de crescimento foi feita segundo a terminologia adotada pelo IAWA COMMITEE (1989).

Em cada secção transversal foram traçados pelo menos 10 raios no sentido casca - medula, ao longo dos quais foram demarcados os limites dos anéis de crescimento. A medição da largura dos anéis de crescimento foi realizada com uma mesa de mensuração Velmex, acoplada a um sistema Quadra-Chek ® 10 , com precisão de $2 \mu \mathrm{m}$. 


\section{Análise dos dados}

A marcação e medição dos limites dos anéis de crescimento seguiu o calendário gregoriano, tendo como início do período vegetativo os meses de agosto/setembro de um ano e tendo como término os meses de junho/julho do ano seguinte (SCHULMAN 1956). Considerando que as coletas foram realizadas nos meses de agosto e setembro de 2011, o ano-base considerado para as análises foi 2010.

As séries temporais foram comparadas dentro e entre árvores (SOLIZ-GAMBOA et al. 2011, MIRANDA 2015) com o auxílio do software COFECHA (HOLMES 1983) e pela análise gráfica dos incrementos correntes em cada série analisada.

Seguindo os procedimentos preconizados por GRISSINO-MAYER (2001) foi utilizada a janela de 50 anos, com sobreposição de 25 anos, que corresponde a um comprimento de sobreposição de duas vezes o tamanho do segmento a ser examinado. Visando a construção de uma cronologia, foi empregado o software ARSTAN (COOK \& HOLMES 1984).

Para a remoção das tendências de crescimento relacionadas aos sinais não climáticos empregou-se uma padronização das séries temporais (COOK \& KAIRIUKSTIS 1990), por meio do ajuste dos dados dos anéis de crescimento com uma função spline cúbica com porcentagem de longitude de série de $67 \%$. Também foi calculado o EPS (Expressed Population Signal), que representa a similaridade entre uma cronologia individual e uma cronologia hipotética replicada infinitamente a partir dos dados dos raios individuais num dado intervalo definido de tempo (LARA et al. 2005, BRIFFA 1995). Um EPS $\geq 0.85$ é dado como significativo, em que reflete um sinal comum para as séries (WIGLEY et al. 1984).

\section{Dados climáticos}

O conjunto de dados referentes às séries históricas (e.g.: precipitação, temperatura) para a área de estudo foi disponibilizado pelo: INMET (Instituto Nacional de Meteorologia); SIMEPAR (Sistema Meteorológico do Paraná); IAPAR (Instituto Agronômico do Paraná) e ANA (Agência Nacional de Águas). Os dados de temperaturas máxima, média e mínima foram obtidos através da Estação Meteorológica de Telêmaco Borba, referentes ao período de 1977 até 2010.

Para a precipitação, as informações que compõe o banco de dados da ANA, por meio do Sistema Hidroweb (http://hidroweb.ana.gov.br/) contempla uma série de estações pluviométricas na região, a qual a estação de Salto Mauá (Estação 2450006) foi a utilizada, por ser a mais próxima da região da pesquisa e contempla 61 anos de medição (1946 a 2006).

Para a série histórica dos pulsos de inundação, foram utilizados os dados de DE FRANÇA (2002), que analisou o regime fluvial do rio Tibagi, em três porções, ao longo do rio (Tabela 1).

Tabela 1. Registros de vazão máxima do Rio Tibagi registradas na Estação Tibagi, para a série histórica de 1932-1995. Adaptado de DE FRANÇA (2002).

Table 1. Records of the Tibagi River maximum flow rates recorded at theTibagi Station, for the1932-1995 historical series. Adapted from DE FRANÇA (2002).

\begin{tabular}{|c|c|c|c|c|c|c|c|c|c|}
\hline $\begin{array}{c}\text { Período } \\
\text { (ano) }\end{array}$ & $\begin{array}{l}\text { Vazão } \\
\left(m^{3} s^{-1}\right)\end{array}$ & $\begin{array}{c}\text { Período } \\
\text { (ano) }\end{array}$ & $\begin{array}{l}\text { Vazão } \\
\left(\mathrm{m}^{3} \mathrm{~s}^{-1}\right)\end{array}$ & $\begin{array}{c}\text { Período } \\
\text { (ano) }\end{array}$ & $\begin{array}{l}\text { Vazão } \\
\left(\mathrm{m}^{3} \mathrm{~s}^{-1}\right)\end{array}$ & $\begin{array}{r}\text { Período } \\
\text { (ano) }\end{array}$ & $\begin{array}{l}\text { Vazão } \\
\left(\mathrm{m}^{3} \mathrm{~s}^{-1}\right)\end{array}$ & $\begin{array}{l}\text { Período } \\
\text { (ano) }\end{array}$ & $\begin{array}{l}\text { Vazão } \\
\left(\mathrm{m}^{3} \mathrm{~s}^{-1}\right)\end{array}$ \\
\hline $1932^{*}$ & 694 & 1947 & 653 & $1970^{\star *}$ & 713 & 1980 & 840 & 1990 & 1154 \\
\hline 1935 & 869 & $1954^{\star \star}$ & 865 & $1971^{* *}$ & 1324 & $1982^{*}$ & 1075 & 1992 & 1023 \\
\hline 1937 & 1435 & $1955^{\star \star}$ & 855 & $1972^{*}$ & 1008 & $1983^{*}$ & 1375 & $1993^{*}$ & 1162 \\
\hline $1941^{*}$ & 655 & $1957^{*}$ & 1048 & $1975^{\star *}$ & 664 & $1987^{*}$ & 1319 & $1994^{*}$ & 768 \\
\hline $1946^{\star *}$ & 735 & 1963 & 721 & 1976 & 704 & $1988^{* *}$ & 852 & $1995^{\star \star}$ & 1378 \\
\hline
\end{tabular}

Legenda: * Anos com registro de El Niño; ** Anos com registro de La Niña.

Objetivando verificar eventuais relações das variáveis climáticas (precipitação acumulada e média da temperatura máxima e mínima) e a largura do anel de crescimento para o ano corrente foram realizadas correlações simples, não paramétricas. Presumindo que os fatores climáticos de anos anteriores podem incidir na atividade cambial e, consequentemente, no crescimento das árvores no ano corrente, também foi realizada análises de correlação referentes ao ano anterior. As análises foram realizadas via software Microsoft Excel 2016.

Para relacionar as larguras dos anéis de crescimento com o fenômeno ENSO, utilizou-se dados provenientes de LI et al. (2011), por meio do website Climate Explorer (https://climexp.knmi.nl/). A mesma base de dados também foi utilizada para gerar as correlações entre a cronologia estabelecida para a 
espécie e a SST (Temperatura da Superfície do Mar), pelo banco de dados hadISST1 (Hadley Centre SST data set), e para os dados de precipitação a nível global, pelo banco de dados CRU TS 3.23.

\section{RESULTADOS}

\section{Anéis de crescimento de $C$. langsdorffii}

$\mathrm{O}$ anel de crescimento de $C$. langsdorffii é macroscopicamente distinto (Figura 2A), sendo este caracterizado pela presença de faixas marginais (com mais de 3 células de largura) de parênquima axial, contendo, em sua maioria, canais axiais intercelulares que delimitam as camadas de crescimento. Foram também encontrados falsos anéis nas amostras, em formato de cunha, bem como anéis descontínuos (Figuras 2B e 2C).

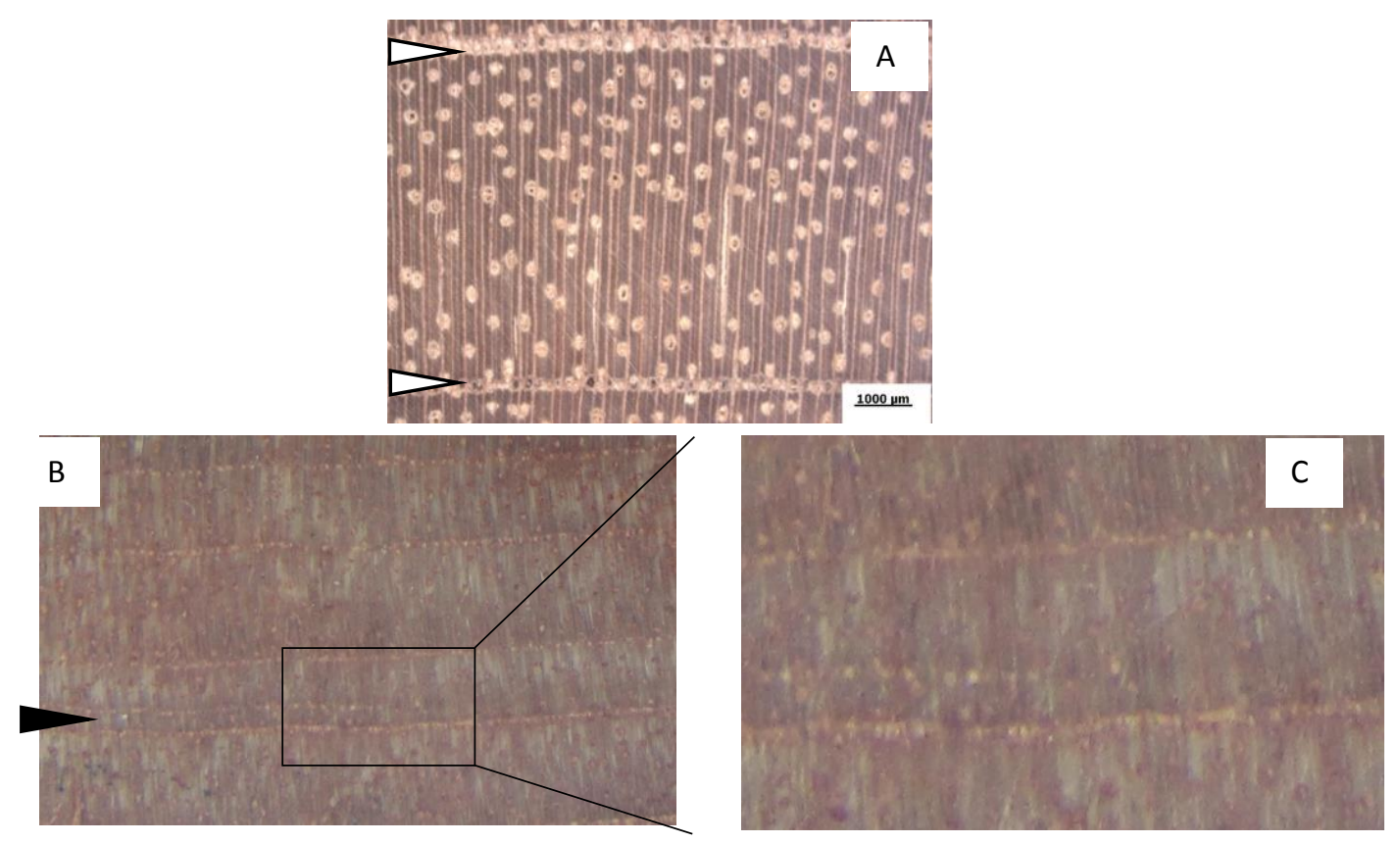

Figura 2. Imagem transversal de Copaiba langsdorffii. Anel de crescimento típico (seta branca), evidenciando a delimitação pela presença de faixas marginais de parênquima axial associada a presença de canais axiais intercelulares (A). Exemplo de anel de crescimento descontínuo (seta preta) (B). Imagem em detalhe (C).

Figure 2. Copaiba langsdorffii transverse image. Typical growth ring (white arrow), showing the boundaries by axial parenchyma in marginal bands associated with the presence of intercellular axial canals (A). Example of discontinuous growth ring (black arrow) (B). Picture in detail (C).

\section{Dendrocronologia e relações climáticas}

De um total de 40 séries radiais amostradas e analisadas nos indivíduos, foram realizados os controles das medições de largura e dos limites dos anéis de crescimento. Desse total, para a sincronização dos dados e estabelecimento da cronologia foram utilizadas 25 séries radiais. $O$ valor de intercorrelação final foi de 0,469 , acima do valor limite estabelecido para o segmento de análise considerado de 50-25 anos $(r=0,3281, p<0,01)$, conforme Tabela 2 .

Tabela 2. Estatística descritiva da cronologia de Copaifera langsdorffii na área de estudo. Table 2. Copaifera langsdorffii descriptive statistics chronology in the studied area.

\begin{tabular}{ll}
\hline \multicolumn{1}{c}{ Sítio } & Telêmaco Borba/PR \\
\hline Número de árvores & 4 \\
Séries temporais radiais (de um total de) & $25(40)$ \\
Anos (Período considerado) & $240(1771-2010)$ \\
Número de anéis analisados & 3550 \\
Intercorrelação & $0,469^{*}$ \\
Segmento de verificação (em anos) & $50 / 25$ \\
Ponto crítico ( $\mathrm{p}<0,01)$ & 0,3281 \\
Sensibilidade média & 0,357 \\
\hline${ }^{*}$ Estatisticamente significativo $(\mathrm{p}<0,01)$. &
\end{tabular}


Assim, conforme a Tabela 2, a cronologia estabelecida apresentou uma série de 240 anos, compreendendo o período entre 1771 a 2010. A sensibilidade média ficou acima do valor de 0,30 $(\mathrm{sm}=0,357)$. Os valores do EPS variaram de 0,60 a 0,92 , tendo como período ótimo (EPS $\geq 0,85)$ a partir de 1880, e o R-bar obteve valor médio de 0,42 (Figura 3). A relação da cronologia da espécie com os picos de vazão do rio Tibagi pode ser visualizada na figura 4.
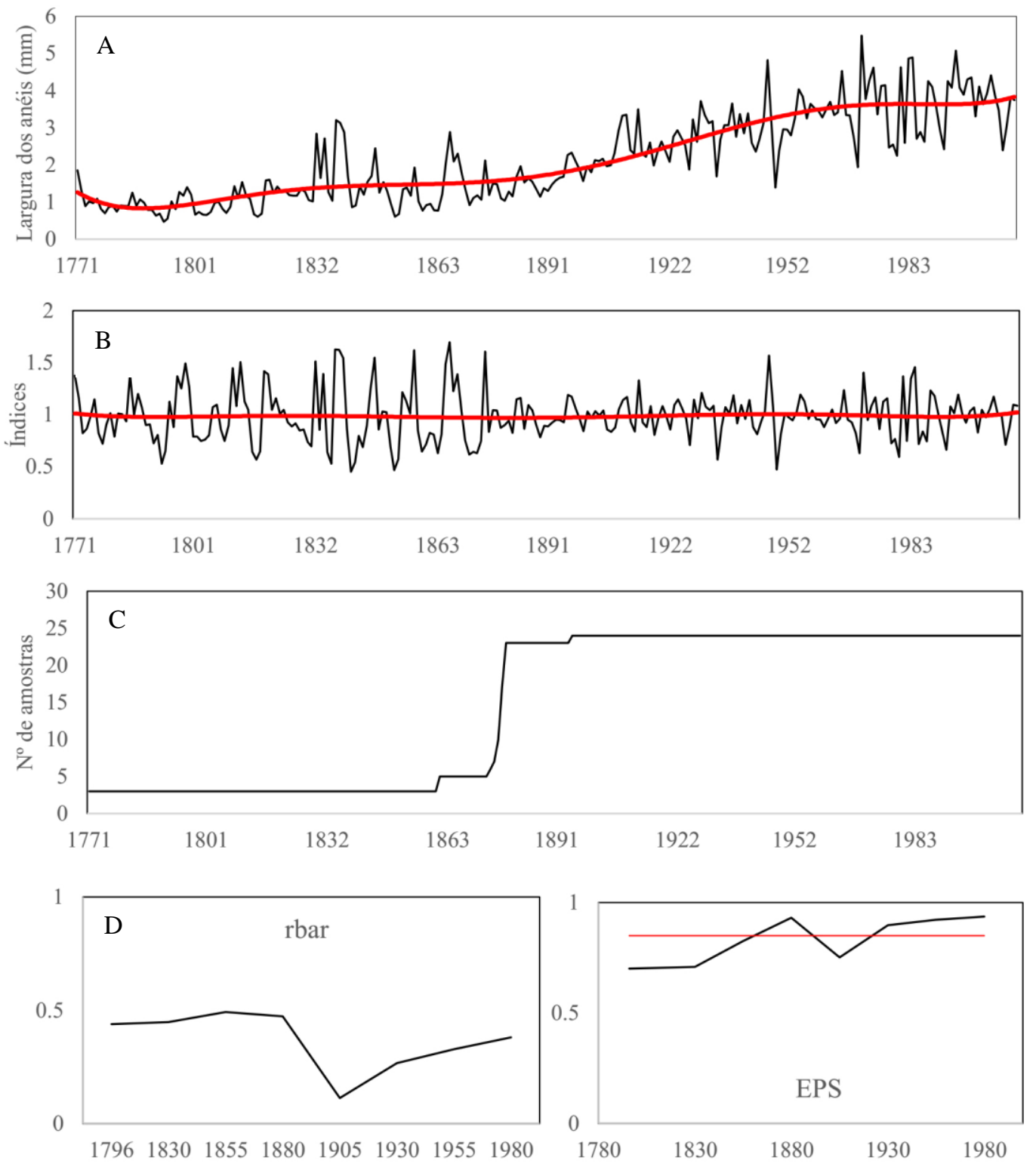

Figura 3. Série bruta de crescimento, com a suavização por índices de spline (A); cronologia standart para a série estabelecida (B); e número de amostras associada a cada ano calendário (C); Valores de R-bar e EPS para cada período analisado (D).

Figure 3. Growth series with smoothing by spline indices $(A)$; standard chronology for the established series (B); and number of samples associated with each calendar year (C); $R$-bar and EPS values for each period (D).

De acordo com a Figura 4, ao analisar a correlação entre os picos de vazão e a cronologia estabelecida com base nesses quatro indivíduos, nota-se que até a década de 70 , os dados não apresentam correlação significativa $(r=-0,25)$, porém após 1970, os valores de correlação aumentam consideravelmente $(r=0,42)$.

Apesar da correlação total ser considerada baixa e não significativa $(r=-0,09 ; p<0,05)$, é possível observar diversos anos em que o crescimento das árvores de $C$. langsdorffii convergem com a curva dos registros de ENSO (Figura 5), como por exemplo nos anos de 1913, 1915, 19181939 e 1983, 1993, 1998, coincidentemente anos de ocorrência do evento El Niño. 


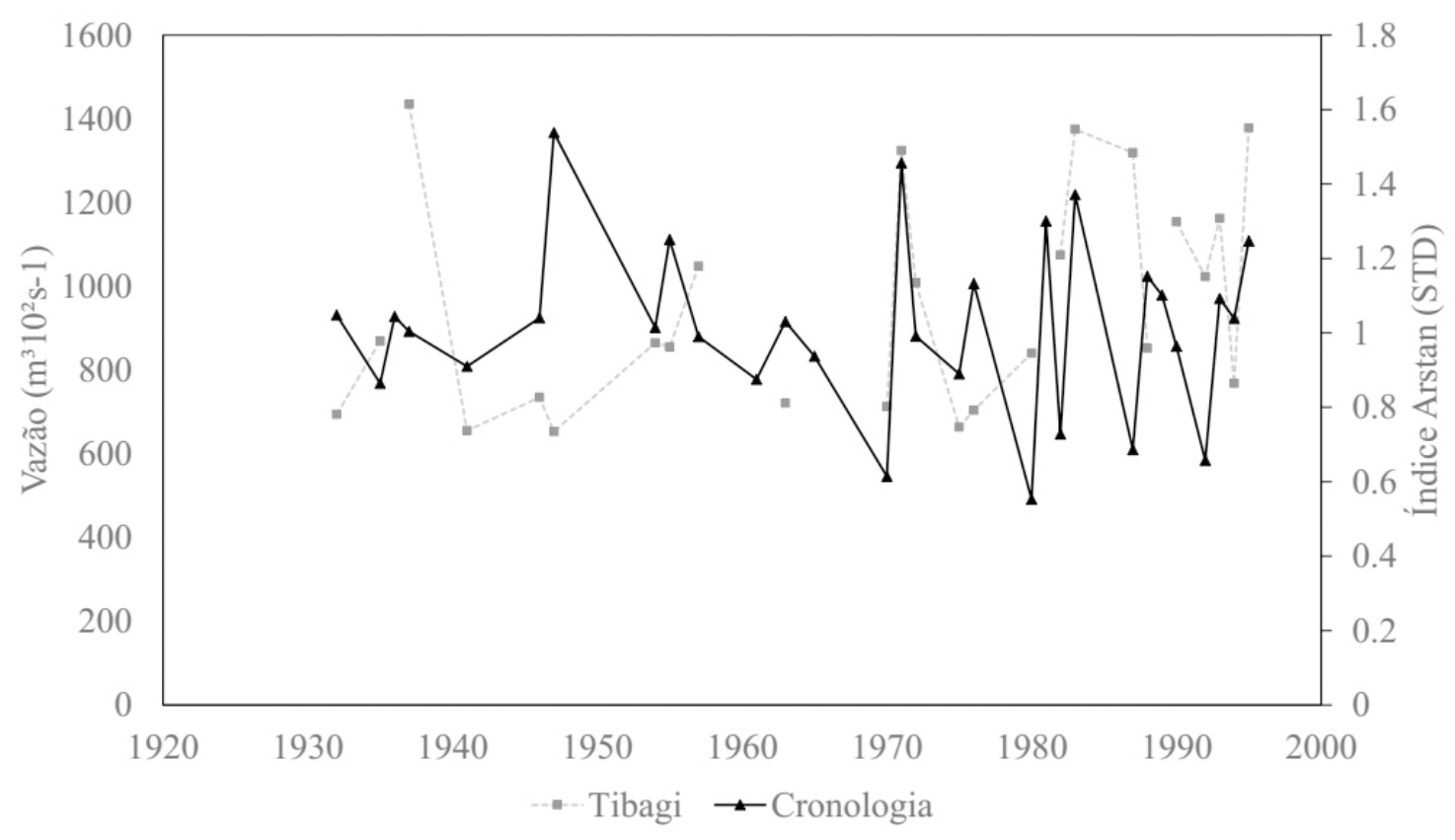

Figura 4. Picos de vazão do Rio Tibagi, relacionados com a cronologia estabelecida para a espécie, com forte correlação a partir da década de 1970.

Figure 4. Tibagi River flow peaks, related to the established chronology, with strong correlations by the 1970s.

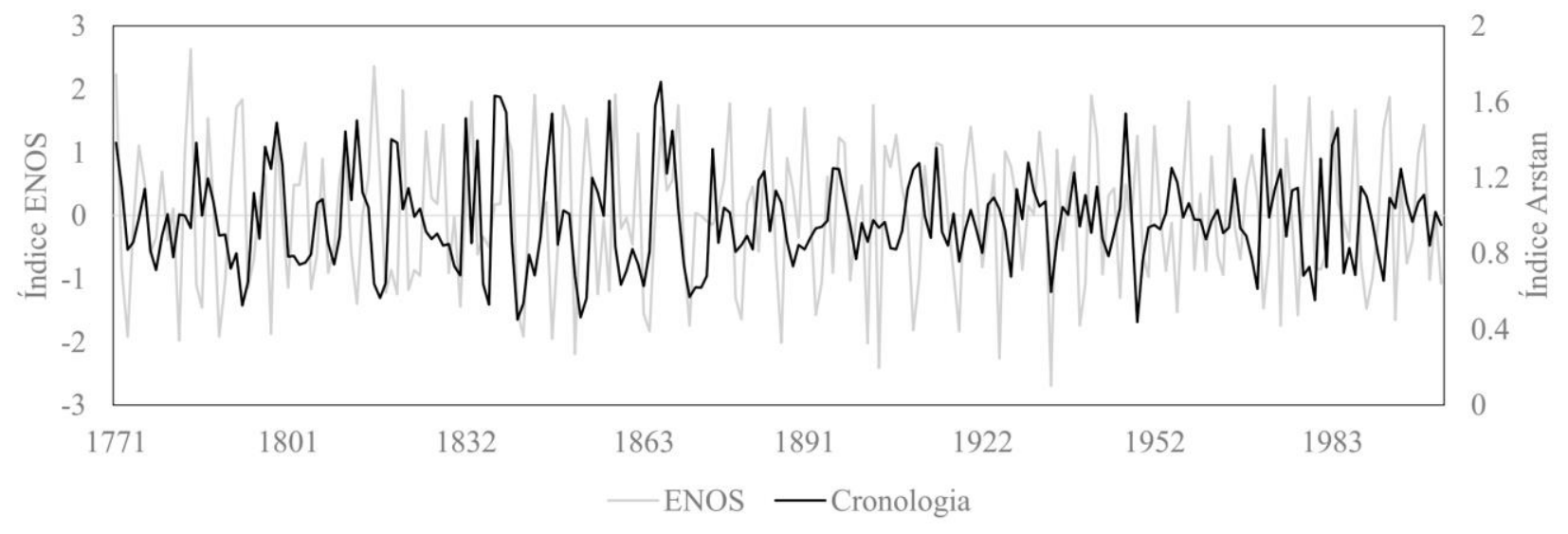

Figura 5. Relação do registro dos últimos 200 anos das variações climáticas do fenômeno ENSO, na América do Sul e a cronologia indexada de crescimento de $C$. langsdorffii.

Figure 5. ENSO events recordings for the last 200 years in South America, and the Copaifera langsdorffii indexed chronology.

As variáveis climáticas de temperatura e precipitação afetaram o crescimento das árvores no período avaliado, de acordo com a Figura 6 . A espécie apresenta correlações significativas positivas $(p<0,05)$ com as temperaturas na estação de crescimento (verão), tanto do ano anterior quanto do ano corrente. Em contrapartida temperaturas altas no início da primavera do ano anterior $(r=-0,39)$ mostram correlações negativas e significativas. Correlações com a precipitação foram positivas no período de crescimento da estação anterior e negativas na estação de crescimento do ano corrente, ambas significativas.

Pode-se observar que as anomalias na temperatura do Oceano Atlântico Central Sul influenciam no padrão de precipitação da região, bem como no desenvolvimento da $C$. langsdorffii, que responde de maneira positiva $(r=0,5)$. As altas temperaturas do Oceano Atlântico Sul, principalmente no verão, tiveram um efeito positivo sobre a cronologia da espécie, conforme Figura 7. 


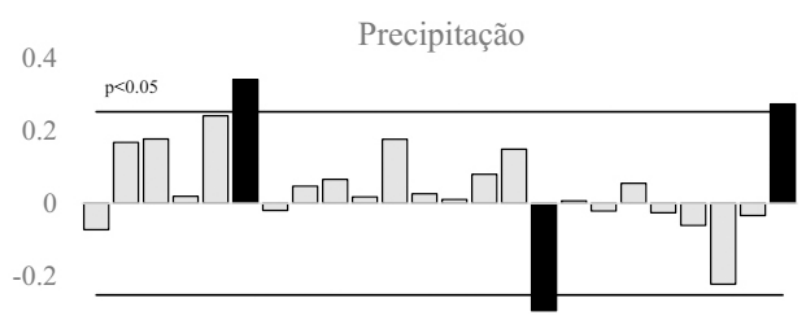

$-0.4$

A S OND J F MAM J J A S OND J F MAM J J Ano anterior

Temperatura média

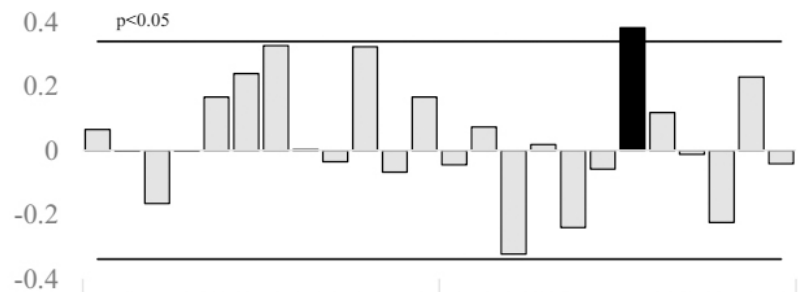

A S O N J F MAM J J A S O N J F MAM J J

Ano anterior
Ano corrente
Ano corrente

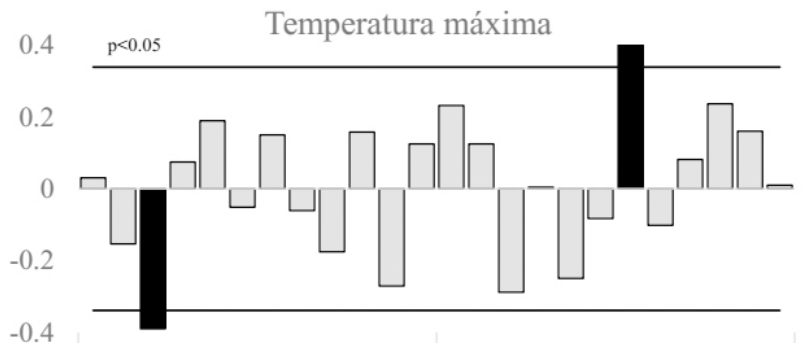

A S O N D J F MAM J J A S O N J F M A M J J

Ano anterior

Ano corrente

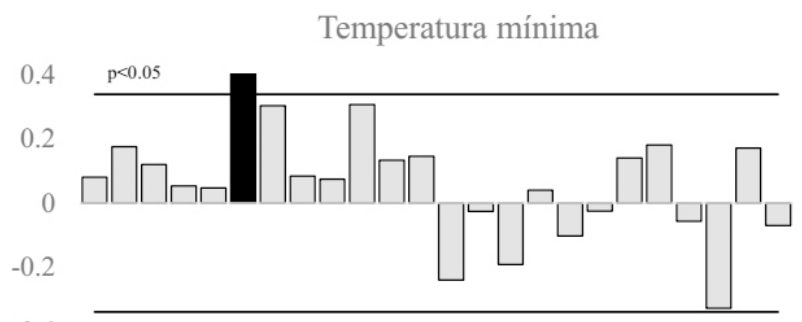

A S O N D J F A M J A S O N D F MAM J J

Ano anterior

Figura 6. Correlações do crescimento com variáveis meteorológicas.

Figure 6. Correlations between growth indices and climate variables.

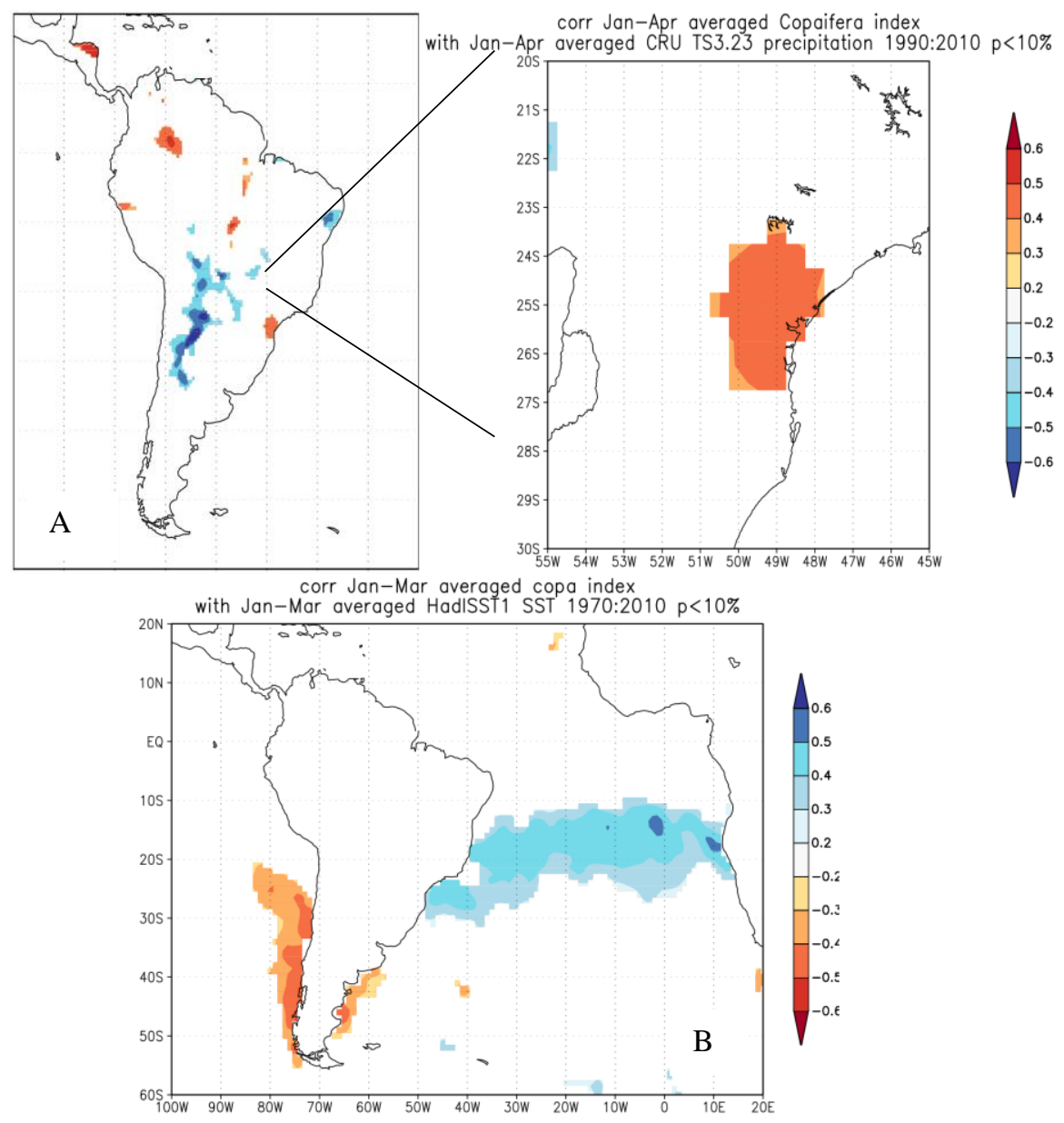

Figura 7. Relações espaciais entre a cronologia de C. langsdorffii e precipitação, para o período 1990-2010 (A). Correlação entre índices de crescimento de $C$. langsdorffii e anomalias de SST (B).

Figure 7. Spacial relations between chronolgy and precipitation, for the1990-2010 period (A). Correlations between growth indices and SST anomalies (B). 


\section{DISCUSSÃO}

Verificou-se a ausência de canais secretores axiais na maior parte do lenho, o que também foi evidenciado nos trabalhos de MARCATI et al. (2011) e MELO JR. et al. (2011). Também foi evidenciado a presença de falsos anéis que podem estar associados a variações ambientais, como secas, no período de crescimento e estresse hídrico (FRITTS 1976, PRYIA \& BHAT 1998, WIMMER 2002, COPENHEAVER et al. 2006). MELO JR. et al. (2011) associam a formação de falsos anéis em C. langsdorffii, na região de Jaguariaíva, PR, aos períodos sucessivos de seca, que alteram o crescimento das árvores e consequentemente o registro da marcação dos limites dos anéis de crescimento. CECCANTINI et al. (2008) afirmam que a grande diversidade de formas que os anéis de crescimento pode assumir está diretamente ligado as condições ambientais em que cada espécie está submetida bem como sua característica anatômica. Portanto, o reflexo das larguras dos anéis de crescimento é verificado por meio da intercorrelação dos raios, em que é possível identificar e eliminar os falsos anéis presentes nas amostras deste presente estudo.

Os valores de intercorrelação encontrados corroboram com alguns trabalhos realizados na mesma região de estudo, para Araucaria angustifolia $(r=0,318)$ e Aspidosperma polyneuron $(r=0,329)$ (ADENESKY-FILHO 2014, LONGHI-SANTOS 2017). Assim, o valor de intercorrelação da série temporal de C. langsdorffii $(r=0,469)$ ficou acima do ponto crítico estatístico, o que indicaria o potencial de sua utilização para análises dendrocronológicas, ecológicas, bem como em reconstruções climáticas regionais.

Árvores que se encontram em condições de planície aluvial, como as deste estudo, geralmente tem suas taxas de crescimento (largura dos anéis de crescimento) afetadas pelo regime hídrico do rio, que pode ser traduzido pelos índices de vazão. Altas taxas de vazão condicionam alterações no crescimento vegetal, podendo gerar estresse hídrico (LARCHER 2000), pela elevação dos níveis freáticos do solo e consequente anoxia, do mesmo modo que baixas taxas de vazão rebaixam o nível freático do solo. As variações da entrada ou saída de nutrientes carregados pelo rio são dependentes do regime hídrico e, portanto, dos eventos climáticos de maior escala.

Durante a década de 1980-1990, as taxas de vazão do rio Tibagi tiveram uma influência positiva na cronologia de $C$. langsdorffii. Neste período, anomalias climáticas foram registradas e relacionadas à influência direta do El Niño sobre a superfície da região Sul do Brasil (GRIMM \& TEDESCHI 2009), e que de maneira direta e indireta refletiram nos níveis de vazão dos grandes rios, entre eles, o Tibagi.

Ainda que os pulsos de inundação apresentem efeito conhecido sobre o desenvolvimento dos vegetais (SCHÖNGART et al. 2004 e 2005, BUDKE 2007), outras variáveis meteorológicas podem estar afetando o desenvolvimento e crescimento das árvores de $C$. langsdorffii, como a temperatura e a precipitação. Em geral, observa-se que houve correlação positiva no crescimento das árvores de $C$. langsdorffii tanto para a variável temperatura quanto para precipitação, que segundo CAVALCANTI et al. (2009) é uma característica esperada para a região sul do Brasil pois, o deslocamento de massa quente e úmida vindas da Amazônia, juntamente com o gradiente sazonal, provocam precipitações nessa região.

Apesar dos invernos na região serem considerados mais secos, podem haver precipitações, que algumas vezes estão associadas aos eventos ENSO do ano anterior, chegando a causar sérias enchentes na região Sul do Brasil (GRIMM et al. 2000, CAVALCANTI et al. 2009). Isso indica que a formação do anel de crescimento pode estar ligada à um estímulo climático, e não apenas ambiental (no caso dos pulsos de inundação do rio).

GRIMM et al. (1998) observaram, para o sudoeste do estado de São Paulo, características comuns com as da região Sul do Brasil, com anomalias positivas no inverno-primavera (junho-dezembro) nos anos dos eventos bem como no outono de anos posteriores. As chuvas, principalmente do ano anterior, indicam que a precipitação do ano anterior tem influência no índice de largura dos anéis de crescimento da espécie. Essa influência ocorre pela formação de reservas de nutrientes, que são mobilizadas na próxima estação de crescimento (HOU 1985, ANDREACCl et al. 2014). LATORRACA et al. (2015) encontrou padrão semelhante, em que a precipitação tanto no ano anterior quanto no ano corrente durante 0 inverno apresentou maior correlação significativa positiva $(r=0,34)$ no crescimento de Schizolobium parahyba. A precipitação também é apontada por alguns autores como uma variável que regula o crescimento dos indivíduos de florestas tropicais secas (SCHÖNGART et al. 2006, ROZENDAAL \& ZUIDEMA 2011, LOCOSSELLI et al. 2016b).

A atuação efetiva dos eventos ENSO são acompanhados por anomalias que ocorrem não só nas regiões do Pacífico como também em todo o mundo. Em anos de El Niño há uma tendência de anomalias positivas de precipitação para o Sul do Brasil (Figura 7), esse evento está associado a diferentes fenômenos, como por exemplo mudanças na circulação atmosférica (PSA- Pacific South American) que se 
estendem desde a área convectiva no Oceano Pacífico até a América do Sul (KOUSKY \& CAVALCANTI 1984, PAEGLE \& MO 2002). Para a cronologia de C. langsdorffii, foram observadas correlações positivas com a precipitação nos últimos 20 anos, para o período de verão e início do outono (Jan-Abr), corroborando os resultados de GRIMM et al. (1998). Por outro lado, estudo realizados na região Central do Brasil, mostra que o regime de chuva é influenciado pelas anomalias de SST do Atlântico Sul e não pelo Pacífico Equatorial (LOCOSSELLI et al. 2016b), mesmo padrão encontrado para região Centro-Leste do Brasil (YOON \& ZENG 2010).

Na Figura 7, pode-se observar a influência das anomalias que ocorrem no Oceano Atlântico, para o período de 1970-2010, sobre o desenvolvimento das árvores de $C$. langsdorffii. A presença de anomalias de SST no Oceano Atlântico influenciam diretamente o clima da região Sul e Sudeste do Brasil (CARDOSO 2005), como por exemplo o surgimento de fortes tempestades e ventos (ciclones extratropicais), como uma resposta da diferença de temperatura do continente e do oceano (GAN \& RAO 1991). As altas temperaturas do Atlântico Sul, atuam de maneira significativa nas flutuações climáticas que ocorrem na região sul do Brasil (CARDOSO 2005, BORMA \& NOBRE 2013). Correlações positivas significativas entre as anomalias de SST e a precipitação também foram observadas por DIAZ et al. (1998) sobre o Uruguai e o Rio Grande do Sul, mais evidenciados nos períodos de dezembro e abril a junho, ou seja, o verão e o início do outono.

\section{CONCLUSÃO}

Os resultados permitiram concluir que Copaifera langsdorffii apresentou anéis de crescimento distintos, demarcados por faixas de parênquima marginal com a presença de canais axiais, com idades alcançando até 240 anos, informação inédita para a espécie na região Sul do Brasil. As cronologias desenvolvidas foram significativas, e tiveram forte sensibilidade para detectar relações com as precipitações, em especial no ano anterior do crescimento, sendo identificada relações inclusive com as taxas de vazão do rio Tibagi e com a temperatura na estação de crescimento (outubro a março). As anomalias de SST, ocorrentes principalmente no Oceano Atlântico Central Sul, apresentam influência significativa no desenvolvimento de $C$. langsdorffii principalmente para o verão e início do outono. Esses resultados reforçam a utilização da espécie para os estudos de reconstruções climáticas e seus efeitos, bem como pode contribuir para o entendimento da dinâmica do clima da região Sul do Brasil.

\section{AGRADECIMENTOS}

Ao IAPAR, SIMEPAR e ao INMET, pela disponibilidade dos dados meteorológicos e séries históricas para a região.

\section{REFERÊNCIAS}

ADENESKY-FILHO E. 2014. Florística, fitossociologia e dendroecologia em encosta e planície do médio rio Tibagi, Telêmaco Borba, Paraná, Brasil. (Doutorado em Engenharia Florestal). Curitiba: UFPR. 140p.

ALVARES CA et al. 2013. Köppen's climate classification map for Brasil. Meteorologische Zeitschrift 22: 711-728.

ANDREACCI $\mathrm{F}$ et al. 2014. Sinais climáticos em anéis de crescimento de Cedrela fissilis Vell. em diferentes tipologias de florestas ombrófilas do sul do Brasil. Floresta 44: 323-332.

BORMA LS \& NOBRE CA. 2013. Secas na Amazônia: causas e consequências. São Paulo: Oficina de Textos. 351 p.

BRIFFA KR. 1995. Interpreting high-resolution proxy climate data: the example of dendroclimatology. In: STORCH H \& NAVARRA A. (Eds.). Analysis of climate variability, applications of statistical techniques. Berlin: Springer. p. 77-94.

BUDKE JC. 2007. Pulsos de inundação, padrões de diversidade e distribuição de espécies arbóreas em uma floresta ribeirinha no sul do Brasil. Tese (Doutorado em Botânica). Porto Alegre: UFRGS. 195p.

CARDOSO AO. 2005. Relações entre a TSM nos Oceanos Atlântico e Pacífico e as condições climáticas nas regiões Sul e Sudeste do Brasil. Tese (Doutorado em Meteorologia). São Paulo: USP. 192p.

CALLADO CH et al. 2014. Studies on cambial activity: advances and challenges in the knowledge of growth dinamics of Brazilian woody species. Annals of the Brazilian Academy of Sciences 85: 277-283.

CARVALHO PER. 2003. Espécies arbóreas brasileiras. Vol. 1. Colombo: Embrapa Florestas. 1040p.

CAVALCANTI IFA et al. 2009. Tempo e Clima no Brasil. São Paulo: Oficina de Textos. 464p.

CECCANTINI GCT et al. 2008. Os anéis de crescimento das árvores: Desvendando as mudanças climáticas. In: BUCKERIDGE MS. (Ed.). Biologia das mudanças climáticas no Brasil. São Paulo: Ed. Rima. p.57-75.

COELHO CAS et al. 2012. Climate diagnostics of three major drought events in the Amazon and illustrations of their seasonal precipitation predictions. Meteorological Applications 19: 237-255.

COOK ER \& HOLMES RL. 1984. Program ARSTAN users manual. Tucson: The University of Arizona Press.

COOK ER \& KAIRIUKSTIS LA. 1990. Methods of Dendrochronology: Applications in the Environmental Sciences. Dordrecht: Kluwer. 394p.

COPENHEAVER CA et al. 2006. Causation of false ring formation in Pinus banksiana: A comparison of age, canopy 
class climate and growth rate. Forest Ecology and Management 236: 348-355.

COSTA MS et al. 2015. Growth analysis of five Leguminosae native tree species from a seasonal semidecidual lowland forest in Brazil. Dendrochronologia 36: 23-32.

CUNHA RG et al. 2011. El Niño/La Niña - Oscilação Sul e seus impactos na agricultura brasileira: fatos, especulações e aplicações. Revista Plantio Direto 20: 18-22.

DIAZ AF et al. 1998. Relationships between precipitation anomalies in Uruguay and southern Brazil and sea surface temperature in the Pacific and Atlantic oceans. Journal of Climate 11:251-271.

DE FRANÇA V. 2002. O rio Tibagi no contexto hidrogeográfico paranaense. In: MEDRI ME et al. (Eds.). A Bacia do Rio Tibagi. Londrina: UEL. p. 45-63.

DEZZEO N et al. 2003. Annual tree rings revealed by radiocarbon dating in seasonally flooded forest of the Mapire River, a tributary of the lower Orinoco River, Venezuela. Plant Ecology 168: 165-175.

FRITTS HC. 1976. Tree rings and climate. Oxford: Elsevier Science. 582p.

GAN MA \& RAO VB. 1991. Surface Cyclogenesis over South America. Monthly Weather Review 119: 1293-1302.

GONÇALVES GV. 2008. Dendrocronologia no Alto São Bento, em Évora: curva de calibração para datações no âmbito da arqueologia. Boletim Cultural "A cidade de Évora" 7. p.73-97.

GRIMM AM et al. 2000. Climate variability in Southern South America associated with El Niño and La Niña. Journal of Climate 13: 35-58.

GRIMM AM \& TEDESCHI RG. 2009. ENSO and Extreme Rainfall Events in South America. Journal of Climate 22: 15891609.

GRIMM AM et al. 1998. Precipitation anomalies in Southern Brazil associated with El Niño and La Niña events. Journal of Climate 11: 2863-2880.

GRISSINO-MAYER HD. 2001. Evaluating crossdating accuracy: A manual and tutorial for the computer program COFECHA. Tree-Ring Research 5: 205-221.

HOLMES LR. 1983. Computer-assisted quality control in tree-ring dating and measurement. Tree-ring Bulletin 43: 69-78.

HOU W. 1985. Seasonal Fluctuation of Reserve Materials in the Trunkwood of Spruce [Picea abies (L.) Karst.]. Journal of Plant Physiology 117: 355-362.

KOUSKY VE \& CAVALCANTI IFA. 1984. Eventos Oscilação Sul - El Niño: características, evolução e anomalias de precipitação. Ciência e Cultura 36: 1888-1899.

IAWA COMMITEE. 1989. IAWA List of Microscopie Features for Hardwood Identification. IAWA Bulletin 10: $219-332$.

IBGE. 2012. Instituto Brasileiro de Geografia e Estatística. Manual Técnico da Vegetação Brasileira. 2.ed. Rio de Janeiro: CRNEA. 271p.

INMET. 2015. Instituto Nacional de Meteorologia. Séries meteorológicas históricas para o Brasil. Disponível em: http://www.inmet.gov.br/portal/index.php?r=bdmep/bdmep. Acesso em: 05 jul. 2015.

INSIDEWOOD. 2004. A web resource for hardwood anatomy. Disponível em: http://insidewood.lib.ncsu.edu/search Acesso em: 26 fev. 2016.

LARA A et al. 2005. Spatial and temporal variation in Nothofagus pumilio growth at tree line along its latitudinal range $\left(35^{\circ} 40^{\prime}-55^{\circ} \mathrm{S}\right)$ in the Chilean Andes. Journal of Biogeography 32: 879-893.

LARCHER W. 2000. Ecofisiologia vegetal. São Carlos: Rima Artes e Textos. 531p.

LATORRACA JVF et al. 2015. Dendrocronologia de árvores de Schizolobium parahyba (Vell.) S. F. Blake de ocorrência na REBIO de Tinguá-RJ. Revista Árvore 39: 385-394.

$\mathrm{LI} \mathrm{J}$ et al. 2011. Interdecadal modulation of El Niño amplitude during the past millennium. Nature Climate Change 1: 114118.

LISI CS et al. 2008. Tree-ring formation, radial increment periodicity, and phenology of tree species from a seasonal semi-deciduous forest in Southeast Brazil. IAWA Journal 29: 189-207.

LOCOSSELLI GM et al. 2016a. Rock outcrops reduce temperature-induced stress for tropical conifer by decoupling regional climate in the semiarid environment. International Journal of Biometeorology 60: 639-649.

LOCOSSELLI GM et al. 2016b. Climate/growth relations and teleconections for Hymenaea courbaril (Leguminosae) population inhabiting the dry forest on karst. Trees-structure and function 30: 1127-1136.

LONGHI-SANTOS T. 2017. Dendroecologia de Aspidosperma polyneuron Müll. Arg. em duas condições geomorfológicas no Sul do Brasil. Tese (Doutorado em Engenharia Florestal). Curitiba: UFPR. 110p.

LORENSI C. 2012. Estudo de eventos de EI Niño e La Niña em anéis de crescimento de árvores para a região sul do Brasil. Dissertação (Mestrado em Meteorologia). Santa Maria: UFSM. 116p.

MAACK R 2012. Geografia Física do Estado do Paraná. 3.ed. Ponta Grossa: UEPG. 440p.

MARCATI CR et al. 2011. Anatomia comparada do lenho de Copaifera langsdorffii Desf. (LeguminosaeCaesalpinioideae) de floresta e cerradão. Revista Brasileira de Botânica 24: 311-320.

MELO JR. JCF et al. 2011. Anatomia ecológica do lenho de Copaifera langsdorffii Desf. (Leguminosae) distribuída em diferentes condições edáficas do cerrado sul-brasileiro. Iheringia Série Botânica 66: 189-200.

MIRANDA BP. 2015. Dendroecologia de Ilex microdonta Reissek e Drimys brasiliensis Miers em dois ambientes altomontanos da Serra do Mar, Paraná, Brasil. Dissertação (Mestrado em Engenharia Florestal). Curitiba: UFPR. 94p.

OLIVEIRA GS. 2001. O EI Niño e você - o fenômeno climático. São José dos Campos: Editora Transtec. 115p.

PAEGLE JN \& MO KC. 2002. Linkages between summer rainfall variability over south America and sea surface temperature anomalies. Journal of Climate 15: 1389-1407.

PRYIA PB \& BHAT KM. 1998. False ring formation in teak (Tectona grandis L.f.) and the influence of environmental 
factors. Forest Ecology and Management 108: 215-222.

RODERJAN CV et al. 2002. As unidades fitogeográficas do estado do Paraná, Brasil. Ciência \& Ambiente 24: 78-118.

ROZENDAAL DMA \& ZUIDEMA PA. 2011. Dendroecology in the tropics: a review. Trees 25: 3-16.

SCHWEINGRUBER FH. 1988. Tree Rings: Basics and Applications of Dendrochronology. Dordrecht: D. Reidel Publishers. 276p.

SCHÖNGART $\mathrm{J}$ et al. 2002. Phenology and stem-growth periodicity of tree species in Amazonian floodplain forests. Journal of Tropical Ecology 18: 581-597.

SCHÖNGART J et al. 2004. Teleconnection between tree growth in the Amazonian floodplains and the El Niño-Southern Oscillation effect. Global Change Biology 10: 683-692.

SCHÖNGART J et al. 2005. Wood growth of Macrolobium acaciifolium (Benth.) Benth. (Fabaceae) in Amazonian blackwater floodplain forests. Oecologia 145: 454-461.

SCHÖNGART $\mathrm{J}$ et al. 2006. Climate-growth relationship of tropical tree species in West Africa and their potencial for climate reconstruction. Global Change Biology 12: 1139-1150.

SCHÖNGART J et al. 2015. Age and Growth Patterns of Brazil Nut Trees (Bertholletia excelsa Bonpl.) in Amazonia, Brazil. Biotropica 47: 550-558.

SCHÖNGART J et al. 2017. Dendroecological Studies in the Neotropics: History, Status and Future Challenges. In: AMOROSO M et al. (Eds). Dendroecology. Tree-Ring Analyses Applied to Ecological Studies. Springer International Publishing. p. 35-73.

SCHULMAN E. 1956. Dendroclimatic changes in Semiarid America. Tucson: University of Arizona Press. 142p.

SOLIZ-GAMBOA CC et al. 2011. Evaluating the annual nature of juvenile rings in Bolivian tropical rainforest trees. Trees 25: 17-27.

STOKES MA \& SMILEY TL. 1968. An introduction to tree-ring dating. Chicago: The University of Chicago Press. 73p.

TOMAZELLO FILHO M et al. 2004. Anatomical features of increment zones in different tree species in the state of São Paulo, Brazil. Scientia Forestalis 66: 46-55.

WIGLEY TML et al. 1984. On the average value of correlated times series, with applications in dendroclimatology and Hydrometeorology. Journal of Climate and Applied Meteorology 23: 201-213.

WIMMER R. 2002. Wood anatomical features in tree-rings as indicators of environmental change. Dendrochronologia 20: 21-36.

WORBES $M$ et al. 2003. Tree ring analysis reveals age structure, dynamics and wood production of a natural forest stand in Cameroon. Forest Ecology and Management 173: 105-123.

YOON JH \& ZENG N. 2010. An Atlantic influence on Amazon rainfall. Climate Dynamics 34: 249-264. 